\title{
Psychophysical scales of the odor oiliness of homologous alcohols
}

\author{
KARL E. HENION 1 \\ THE UNIVERSITY OF TEXAS AT AUSTIN
}

In determining the relative oiliness of an alcohol, a category, a ratio estimation, and three magnitude scales were constructed from the observations of 12 Os who judged the oiliness of $a \quad C_{2}-C_{10}$ series of homologous alcohols. Oiliness was found to be monotonicly increasing on $\left[C_{2}, C_{8}\right]$, after which it declines, octanol being the oiliest. Ratio scales were stable and resemble power functions with exponents of 0.624 to 0.784 on this interval. The oiliness attribute has a short subjective range of $2.5 / 1$, which is consistent with the tendency toward short ranges of olfactory attributes, generally. Oiliness across the series shows only slight evidence of being prothetic.

Research interest in the psychophysics of odor oiliness arises from the possibility that this attribute may affect adaptation to smell (Mozell \& Pffafmann, 1954; Engen, 1964). Homologous alcohols are suitable stimuli for studying this attribute, since there is some evidence that the oiliness of a series of these compounds apparently varies with the number of carbon atoms in the series, i.e., the chain length. Oiliness may then be studied as a function of those physical properties that correlate with chain length. The evidence comes chiefly from the introspective analyses of Pilgrim and Schutz (1957) and of Engen (1962). The latter analysis was part of a larger study of the factor analysis of similarity judgments of a $C_{3}$ to $C_{8}$ alcohol series. The results of the larger study, combined with the conclusion of the introspective analysis, indicated that oiliness may have been the extracted factor that varied with chain length.

The purpose of the present investigation was to make direct psychophysical estimates of the oiliness of a somewhat larger series of homologous alcohols as a follow-up to the introspective studies. The paper also presents the subjective range of the oiliness of the series.

\section{Observers}

\section{METHOD}

Twelve undergraduates, including two women, volunteered as Os without pay; only one had had experience in olfactory experiments.

\section{Odorants}

These consisted of nine undiluted n-aliphatic homologous alcohols from ethanol $\left(C_{2}\right)$ to decanol $\left(C_{10}\right)$. Seven of the nine alcohols available to $E$ were of extremely high purity (viz, chromatographic or Baker-analyzed reagent grades), and the other two were only slightly less pure. Redistillation was not attempted, since the larger distillation columns of the source companies, Baker and Mathieson, Coleman \& Bell, produce an alcohol with a higher level of purity than do the smaller columns in laboratory redistillation.

\section{Procedure}

The experiments were conducted in an air-conditioned and ventilated room, where the temperature was maintained at about $25^{\circ} \mathrm{C}$. $\mathrm{O}$ sniffed Q-tipped cotton saturated with odorant, $1.5 \mathrm{cc}$ of which were kept in its $10 \times 75 \mathrm{~mm}$ Pyrex test tube stopped with an aluminum foil-wrapped cork; when not in use, the cotton was positioned just above the surface of the odorant.

There were five experimental conditions: Three of these required $O$ to make magnitude estimations with different standards, one to make ratio estimations and one to make category ratings. $O$ was exposed to all conditions, each of which he received in a 1 h session, at a rate of one condition per day over 5 days, in most cases consecutive days, and in a counterbalanced order.

The $\mathrm{O}$ was asked to judge the oiliness of the odorant and to ignore all other attributes, to consider each comparison stimulus independently of the others, to sniff the same way on each trial, and to place the cotton at the same distance from his nose. Before the experiment began, each $O$ had 21 -h practice sessions that were held at the same hour on consecutive days. During the sessions, $O$ became familiar with the sniffing procedures to be used in the experimental sessions and had time to develop a satisfactory interpretation of the meaning of the term, oiliness, and the attribute it stands for. The characteristic qualities of heptanol, which is the standard for oiliness in the matching-standard method of Schutz (1964), and the other oilier alcohols in the series were aids in orienting $O$ at the outset to the general idea of oiliness. E stressed in his instructions the multidimensional nature of the stimuli and asked $O$ to be careful to isolate the dimension of interest, i.e., oiliness, from other attributes, like pleasantness or intensity. Relevant examples of multidimensionality were given, such as the variations in pitch and loudness that occurs when the wave frequency of sound stimuli is varied.

The standard for a magnitude estimation condition was butanol, heptanol, or nonanol. $O$ was instructed to let 10 represent the oiliness of the standard, with which he was asked to become familiar. $\mathrm{E}$ presented the nine comparison stimu! singly, including the standard, and it random sequence with five trials per stimulus. O's task was to judge the oiliness of each by assigning a number to it proportionate on a ratio scale to the 10 of the standard. $O$ could refer to the standard as often as desired, provided that between sniffs there was at least a pause of $45 \mathrm{sec}$, which was the minimal time interval required between any two sniffs in the experiment. Before and after each sniff in all conditions, $O$ turned away, took a deep breath of fresh air through his mouth, and exhaled strongly through his nose. Duplicate sets of comparison stimuli were used in all conditions to reduce the possibility of recognition of previously presented stimuli from accidental markings on corks or test tubes.

In the ratio estimation condition, $\mathrm{E}$ presented once, singly, and in irregular order, each of the 36 pairs possible from the nine comparison stimuli. Half of the Os (every other one) began at one end of the order, and the other half at the other end. $O$ 's task was to sniff the left member of a pair first, then the right one, and to report which was the oilier and also what percentage the less oily one was of the more oily one. Each comparison stimulus appeared equally often on the left as on the right.

In the category estimation condition, there were two standards: $O$ was instructed to denote the oiliness of ethanol as 1 and that of decanol as 5. O's task was to assign to a given comparison stimulus an integer from 1 to 5 , inclusive, that reflected the oiliness of the stimulus on an equal-interval scale. As in each of the magnitude estimation conditions, there were 45 presentations of the nine comparison stimuli: singly presented in random sequence and five trials each. $O$ could refer to standards as often as desired. 
Table 1

Scale Values of the Oiliness of Homologous Alcohols by Three Direct Methods

\begin{tabular}{cccc}
\hline & \multicolumn{3}{c}{ Method } \\
\cline { 2 - 4 } $\begin{array}{c}\text { Chain } \\
\text { Length }\end{array}$ & $\begin{array}{c}\text { Magnitude } \\
\text { Estimation }\end{array}$ & $\begin{array}{c}\text { Ratio } \\
\text { Estimationb }\end{array}$ & $\begin{array}{c}\text { Category } \\
\text { Ratingc }\end{array}$ \\
\hline$C_{2}$ & 4.51 & 0.57 & 1.98 \\
$C_{3}$ & 4.75 & 0.54 & 1.85 \\
$C_{4}$ & 6.07 & 0.82 & 2.53 \\
$C_{5}$ & 7.42 & 1.03 & 3.00 \\
$C_{6}$ & 7.63 & 1.29 & 3.46 \\
$C_{7}$ & 9.01 & 1.35 & 3.43 \\
$C_{8}$ & 10.86 & 1.41 & 3.45 \\
$C_{9}$ & 8.38 & 1.30 & 3.46 \\
$C_{10}$ & 7.92 & 1.24 & 3.33 \\
\hline
\end{tabular}

$a$ adjusted and combined

$b$ unadjusted

$c$ arithmetic means

\section{RESULTS}

Geometric means of the 60 numbers assigned by $O s$ to each alcohol were computed for each magnitude estimation condition. The geometric means for the three magnitude conditions were then combined, in order to get data for a more generalized function (Stevens, 1956), using nonanol as the middle standard and not heptanol (discussed below). The results are presented in the first column of Table 1; Engen and Lindström (1963) have described the computational procedure. The separate and combined magnitude estimation scales form functions that are monotonicly increasing on the closed interval $\left[\mathrm{C}_{2}, \mathrm{C}_{8}\right]$. The functions reach a maximum at the $C_{8}$ alcohol and thereafter decrease. On $\left[\mathrm{C}_{2}, \mathrm{C}_{8}\right]$ the magnitude scales resemble power functions: $O$ n this interval the functions plotted in log-log coordinates are linear, with least-squares regressions producing correlation coefficients in the range 0.92 to 0.98 . The power functions for the three scales, with standards of $\mathrm{C}_{4}, \mathrm{C}_{9}$, and $\mathrm{C}_{7}$, and for the combined scale have exponents of 0.624 , $0.635,0.652$, and 0.637 , respectively.

Geometric means of the 12 ratio estimates made by Os for each of the 36 stimulus pairs were computed for the ratio estimation condition, and these means

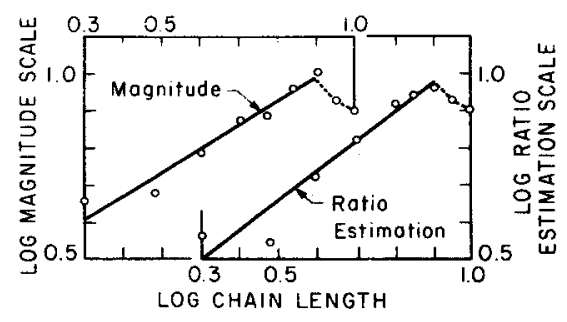

Fig. 1. Log adjusted ratio estimation scale and log combined magnitude estimation scale as functions of $\log$ chain length. were analyzed by the matrix procedure of Ekman (1958). The resulting ratio estimation scale values are shown in the second column of Table 1. Also monotonicly increasing on $\left[\mathrm{C}_{2}, \mathrm{C}_{8}\right]$ and reaching a maximum at $\mathrm{C}_{8}$, this scale resembles a power function on this interval with an exponent of 0.784 . The oiliness range is $2.4 / 1$ for the combined magnitude scale, and $2.6 / 1$ for the ratio estimation scale.

Ratio estimation scale values were proportionally adjusted to the geometric means of the magnitude scale that had nonanol as the standard, in order to compare the adjusted ratio estimation scale with the combined magnitude estimation scale. The two scales are compared in Fig. 1, where they are presented as functions of chain length in $\log$ coordinates. Least-squares regression lines were fitted to these data on $\left[\mathrm{C}_{2}, \mathrm{C}_{8}\right]$, and the results on this interval are linear with correlation coefficients over 0.95 . The two scales are in general agreement, with the slope of 0.783 for the adjusted ratio estimation scale somewhat higher than that of 0.637 for the combined magnitude scale. Engen and Lindström (1963) found the slope of the former type of scales to be somewhat lower than that of the latter type scale in the intensity scaling of amyl acetate.

Of the 36 stimulus pairs of the ratio estimation condition, there are, for example, four pairs such that the ratio of the carbon numbers of the members of each pair is in a ratio of $2 / 1$, viz, 10/5, 8/4, etc. Altogether, the 36 pairs have carbon numbers consisting of 26 different ratios, with most of the stimulus pairs being represented by only one carbon number ratio. Corresponding to these 26 ratios were the 36 subjective ratios made by Os. These two sets of ratios were analyzed in a $\log -\log$ regression plot shown in Fig. 2 . The results indicate internally consistent data, since the least-squares line, which fits the data to a moderate degree $(r=0.68)$, passes through the origin with very small error, generating an equation of $\mathrm{R}=0.941 \mathrm{~S}^{0.711}$.

Mean category ratings are presented in the last column of Table 1 .

\section{DISCUSSION}

These results confirm the conclusions of the introspective analyses previously mentioned as to the generally higher level of oiliness shown by alcohols with longer chain lengths over those with shorter lengths. The result that octanol would turn out to be the oiliest of the alcohols, which both types of ratio scales demonstrated, was not indicated in the early analyses or

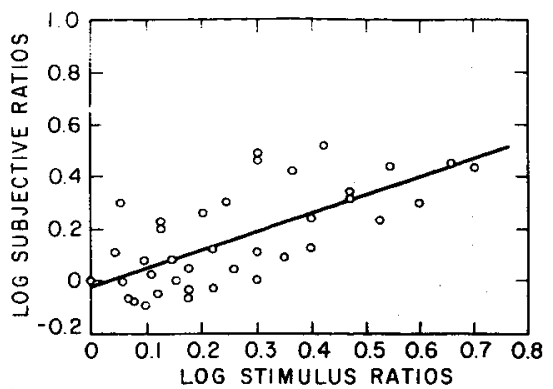

Fig. 2. Log subjective ratios as a function of $\log$ stimulus ratios of carbon numbers.

expected at the outset of the present investigation. It was thought that decanol would be judged the oiliest. Under the assumption of a monotonicly increasing oiliness sequence over the entire $\left[\mathrm{C}_{2}, \mathrm{C}_{10}\right]$ interval studied, it was also thought that heptanol would be a suitable middle standard for generalizing the separate magnitude scales into a combined scale. But heptanol was found to be oilier than its ascendent neighbor in the series, nonanol, and hence the latter was used as the middle standard in the calculations. Although heptanol was a good choice to represent the oiliness attribute in the matching standards method of Schutz (1964), perhaps octanol would have been even a better selection.

Why both ratio scales decline after octanol is far from clear. Judging the oiliness of these alcohols was not a necessarily unpleasant task for $O$, save perhaps for judging nonanol and decanol, which tend to be slightly noisome. For example, one $O$ said that decanol smelled like a stink bug. Some amount of unpleasantness may have made it relatively harder for $\mathbf{O}$ to isolate the attribute of interest in the case of these two alcohols, and hence led him to report less of it present by assigning lower numbers to them.

The undiluted shorter chain alcohols are relatively more intense (Kruger, Feldzamen, \& Miles, 1955; Engen, 1965) and, at the same time, less oily than the larger chain ones, but this inverse relationship did not appear to prevent Os from making satisfactory judgments of oiliness. Os seemed to be able to isolate and judge the oiliness of the shorter ones, since the oiliness range over the entire C-interval studied was only about $2.5 / 1$. Had practically no oiliness been reported by Os as a group for some of the shorter chain alcohols, one would have suspected the range over $\left[\mathrm{C}_{2}, \mathrm{C}_{10}\right]$ to have been considerably higher than it was found to be.

The subjective range apparently is also short for the odor intensity of undiluted 


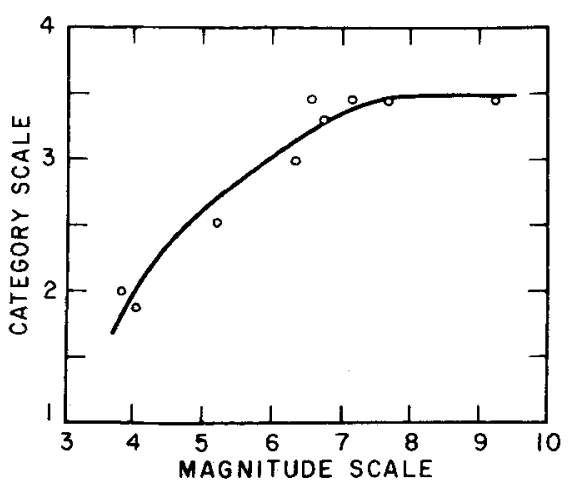

Fig. 3. Category scale as a function of combined magnitude estimation scale. (Fitted by eye.)

homologous alcohols. This is the inference one might make from the adult observations reported by Engen (1965) in an intensity study of homologous alcohols, involving both human infants and adults. The ratio of the geometric mean of adult magnitude estimations of $100 \%$ concentration of propanol to the corresponding geometric mean for $100 \%$ octanol, alcohols representing short and long chains, respectively, seems to be under $1.1 / 1$. Thus, the evidence continues to accumulate that, save possibly for the larger hedonic range (Engen \& McBurney, 1964), olfactory attributes tend to have rather short subjective ranges contrasted to the attribute ranges of other sense modalities.

Perhaps it is only a coincidence, but there is a rough similarity between the oiliness judgments by adults of alcohols on the $\left[C_{7}, C_{10}\right]$ interval in the present investigation and the disruptive breathing responses of human infants to the same subset of alcohols in Engen's intensity study. In both studies, a relative maximum on this subinterval occurred for each of the respective attribute functions at the octanol abscissa, with the functional values for the $C_{7}$ and $C_{10}$ alcohols in the same relative positions, although the infant study involved a deleted interval, the $C_{9}$ alcohol being unavailable. And this similarity obtains in the oilier part of the domains of the oiliness and intensity functions, the part of special interest in the present investigation; however, the similarity does not hold for the oily-like $\mathrm{C}_{6}$ alcohol.

The construction of a category scale was attempted to see if oiliness over the domain $\left[\mathrm{C}_{2}, \mathrm{C}_{10}\right]$ showed prothetic or metathetic properties. The occurrence of almost exactly the same category scale values for the oilier alcohols, taken together with the ratio scaling result that octanol was the oiliest of the alcohols, suggests why the category scale used was not an entirely satisfactory way for Os to discriminate oiliness. Had octanol been chosen for Position 5 on the scale instead of decanol, the discrimination would probably have been better. With this qualification, the category scale is presented in Fig. 3 as a function of the combined magnitude scale. The concave downward relationship implies that oiliness is prothetic. Consistent with this implication is the fact that the category scale was more concave downward than the magnitude scale in a plot, not shown, of both scales as functions of chain length. The implied prothetic property of the oiliness continuum is quite tentative, given the qualification previously mentioned and the fact that, while many of the odorants had an inherent oily character, they had varying qualities.

\section{REFERENCES}

EKMAN, G. Two generalized ratio scaling methods. Journal of Psychology, 1958, 45, 287-295.

ENGEN, T. The psychophysical similarity of the odors of aliphatic alcohols. Report No. 127 1962, University of Stockholm, Psychological Laboratory.

ENGEN, T. Psychophysical scaling of odor intensity and quality. Annals of the New York Academy of Sciences, 1964, 504-516.

ENGEN, T. Psychophysical analysis of the odor intensity of homologous alcohols. Journal of Experimental Psychology, 1965, 70, 611-616. ENGEN, T., \& LINDSTRÖM, C. O. Psychophysical scales of the odor intensity of amyl acetate. Scandinavian Joumal of Psychology, 1963, 4, 23-28.

ENGEN, T., \& McBURNEY, D. H. Magnitude and category scales of the pleasantress of odors. Joumal of Experimental Psychology, $1964,68,435-440$.

KRUGER, L., FELDZAMEN, A. N., \& MILES, W. R. Comparative olfactory intensities of the aliphatic alcohols in man. American Jourral of Psychology, 1955, 68, 386-395.

MOZELL, M. M., \& PFAFFMANN, C. The afferent neural processes in odor perception. Annals of the New York Academy of Sciences, 1954, 58, 96-108.

PILGRIM, F. J., \& SCHUTZ, H. G. Measurement of the qualitative and quantitative attributes of flavor. In: Chemistry of natural food flavors. Quartermaster Food Container Institute, 1957. Pp. 47-55.

SCHUTZ, H. G. A matching standards method for characterizing odor qualities. Annals of the New York Academy of Sciences, 1964, 116, 517-526.

STEVENS, S. S. The direct estimation of sensory magnitudes-loudness. American Joumal of Psychology, 1956, 69, 1-25.

NOTE

1. Address: The University of Texas at Austin, Austin, Texas 78712 .

(Accepted for publication September 30, 1969.) 